\title{
Successful Treatment with Hepatic Arterial Infusion Chemotherapy in a Breast Cancer Patient with Multiple Liver Metastases Who Declined Systemic Therapy
}

\author{
Takaaki Masuda $^{a}$ Osamu Niizeki ${ }^{b}$ Takashi Niizekic Kenji Fujiyoshid ${ }^{c}$ \\ Yuki Ando ${ }^{a}$ Hiroshi Niizeki $^{b}$ Koshi Mimoria \\ aDepartment of Surgery Kyushu University Beppu Hospital, Beppu, Japan; ${ }^{b} N i i z e k i$ Internal \\ Medicine Clinic, Hita, Japan; 'Division of Gastroenterology, Department of Medicine, \\ Kurume University School of Medicine, Kurume, Japan; ${ }^{d}$ Fujiyoshi Breast Clinic, Beppu, Japan
}

\section{Keywords}

Hepatic arterial infusion chemotherapy · Breast cancer - Liver metastasis · Nutrition · Inflammation

\begin{abstract}
Despite improvements in systemic medical therapy (ST), liver metastases (LMs) are a poor prognostic factor in metastatic breast cancer (MBC) patients. We describe a MBC patient with predominant LMs treated with hepatic arterial infusion chemotherapy (HAIC) who declined ST. Moreover, we assessed general health status during treatment using C-reactive protein (CRP)/albumin ratio (CAR) and peripheral platelet count $\times$ CRP multiplier (P-CRP), well-known indicators of systemic inflammatory response. A 64-year-old woman who underwent a total mastectomy with axillary lymph node dissection for an HR-positive, HER2-negative infiltrating ductal BC developed multiple liver, lung, lymph node, and bone metastases. She received ST including paclitaxel plus the anti-vascular endothelial growth factor antibody, bevacizumab, hormone therapy with high-dose toremifene, the oral 5-fluorouracil derivative, $\mathrm{S}-1$, and eribulin. She then declined ST because of the toxicity or decreased treatment motivation thereof, and opted for HAIC with 5FU plus epirubicin followed by Taxane for 1 year and 1 month. Computed tomography revealed a partial response or stable disease in the liver and slow progression in other sites without symptoms or side effects and decreased CEA and CA15-3 levels. The CAR and P-CRP remained low. She survived for 1 year and 3 months after the start of HAIC. This case reveals that HAIC may be an option for advanced BC patients with LMs who cannot receive ST.
\end{abstract}




\section{Introduction}

Despite improvements in systemic medical therapy (ST), metastatic breast cancer (MBC) remains incurable. Greater than one-half of patients with MBC develop liver metastases (LMs) during treatment [1]. LM is a poor prognostic factor in MBC patients, with extensive LMs directly associated with hepatic failure, causing death. The median overall survival after LM diagnosis is between 14 and 29 months [2]. Thus, control of LM may prolong survival. In MBC, including LM, systemic rather than local therapy is the established standard of care; however, ST toxicity is frequently dose-limiting or decreases treatment motivation, especially after long-duration, continuous therapy with multiple regimens. Indeed, nutrition, inflammation, and immune status (systemic inflammatory response [SIR] [3]) impact tumor progression and therapeutic response [4].

Hepatic arterial infusion chemotherapy (HAIC) provides direct delivery of chemotherapeutic agents into tumor-feeding vessels in the liver and minimizes systemic toxicity by keeping systemic exposure to drugs low, leading to improved efficiency in LM [5]. Furthermore, combined HAIC and ST are reported to achieve the therapeutic effect for patients who have progressed despite various prior ST [6]. We describe a MBC patient with predominant LMs treated with HAIC who declined ST. General health status was assessed during treatment using C-reactive protein (CRP [mg/dL])/ albumin $(\mathrm{g} / \mathrm{dL})$ ratio $(\mathrm{CAR})$ and peripheral platelet count $\times$ CRP multiplier (P-CRP), well-known indicators of SIR and prognostic markers for various malignancies [7, 8].

\section{Case Presentation}

In 1999, a 46-years-old woman underwent a total mastectomy with axillary lymph node dissection for an HR-positive, HER2-negative infiltrating ductal BC (pT2a, pN1a, and M0). She received adjuvant chemotherapy (doxifluridine/cyclophosphamide) followed by tamoxifen hormone therapy; however, she discontinued the hormone therapy for approximately 1 year. In 2009, she was treated for axillary lymph node metastases with hormone therapy using the aromatase inhibitor, letrozole. In June 2013, she changed to hormone therapy with fulvestrant due to LMs. In April 2015, she discontinued hormone therapy again, despite efficacy and no side effects.

In April 2017 (Day 0), at 64-years-old, she was referred to our hospital for advanced BC with multiple liver, lung, lymph node, and bone metastases. We recommended ST and she received 3 courses of paclitaxel plus the anti-vascular endothelial growth factor antibody, bevacizumab, resulting in a partial response of metastases in all sites based on computed tomography (CT). She was changed to hormone therapy with high-dose toremifene and the oral 5-fluorouracil derivative, S-1, until CT confirmed progressive disease (PD). Next, she was treated with eribulin, discontinued after 1 course due to general fatigue (Fig. 1A).

She declined ST, including hormone therapy, after 1 course of fulvestrant because of general fatigue and decreased motivation for treatment, although laboratory testing and vital signs were near-normal. The SIR status CAR and P-CRP were also low, suggesting good nutrition, low inflammation status, and normal immunity (Fig. 2). Thus, we proposed terminal care or HAIC as her next treatment.

She opted for HAIC, injected via the proper hepatic artery (Fig. 1A). Beginning on Day 454 , she underwent HAIC with 5FU (1,250 mg/body) plus epirubicin (30 mg/body). CT revealed a partial response or stable disease in the liver and slow progression in other sites without symptoms or side effects and decreased CEA and CA15-3 levels until Day 698 (Fig. 1B, C). The CAR and P-CRP remained low (Fig. 2); however, on Day 803 CT showed PD (lung, liver, lymph nodes, and bone metastases with pleural effusion) and increased tumor marker levels. 
Masuda et al.: Hepatic Arterial Infusion Chemotherapy for Breast Cancer with Liver Metastases

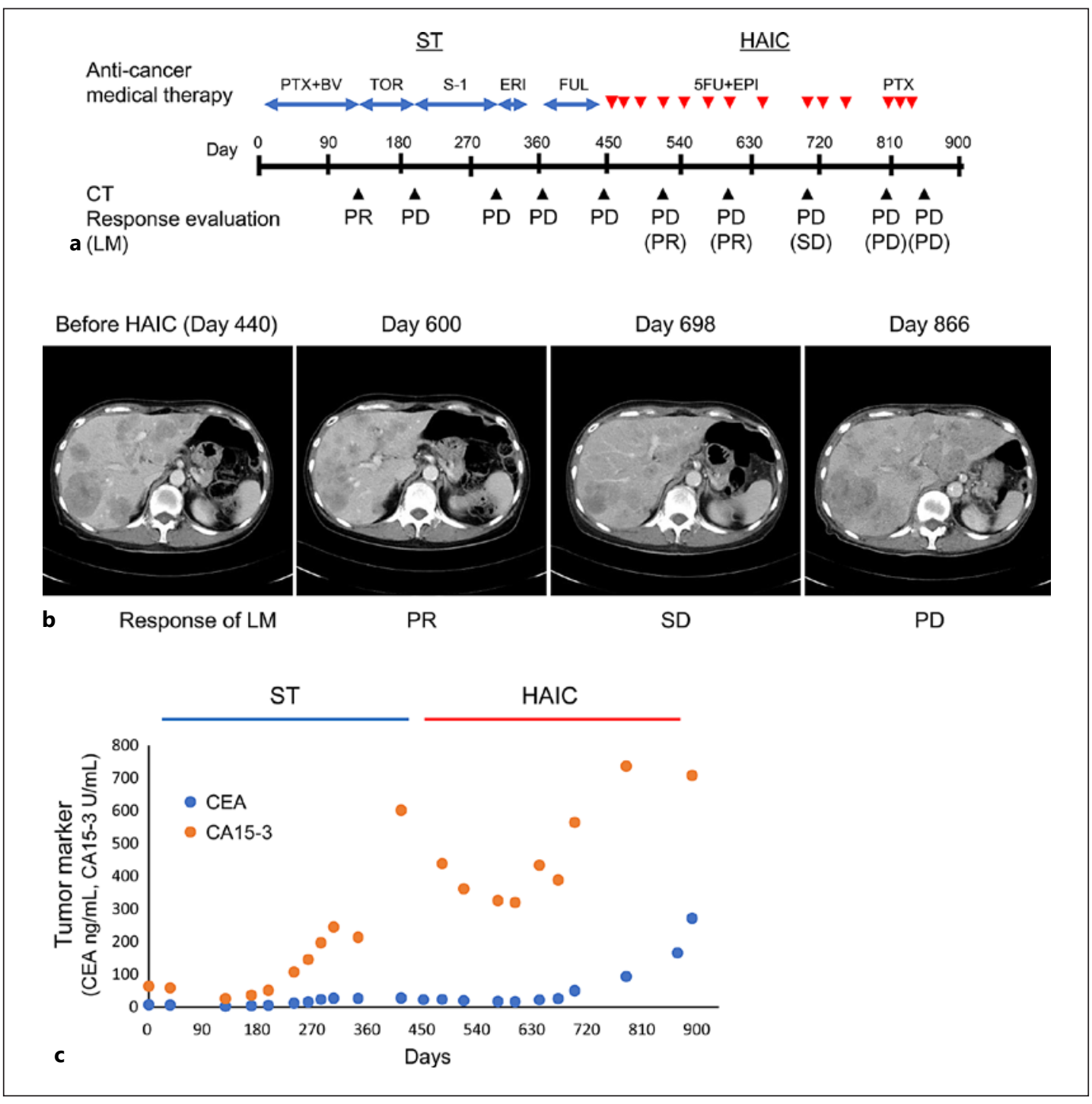

Fig. 1. Treatments and clinical course. A Therapeutic course. B CT. C Tumor markers (CEA and CA15-3). ST, systemic medical therapy; HAIC, hepatic arterial infusion chemotherapy; PTX, paclitaxel; BV, bevacizumab; TOR, toremifene; ERI, Eribulin; FUL, Fulvestrant; EPI, epirubicin; CT, computed tomography; PR, partial response; PD, progressive disease; SD, stable disease; LM, liver metastasis.

She was changed to HAIC with paclitaxel (60 mg/body), resulting in PD on Day 866 (Fig. 1B, C). As shown in Figure 2, CAR and P-CRP increased drastically. She was then admitted to the clinic for general fatigue and a bad cough with jaundice and switched to terminal care. She died on Day 902, 1 year and 3 months after the start of HAIC. She survived for 6 years and 3 months after development of LMs.

\section{Discussion and Conclusion}

We sometimes encounter advanced BC patients who cannot continue ST because of the toxicity or decreased treatment motivation thereof even if the laboratory data are tolerated. However, LM is a life-threatening disease to be cared for in BC [9]. 


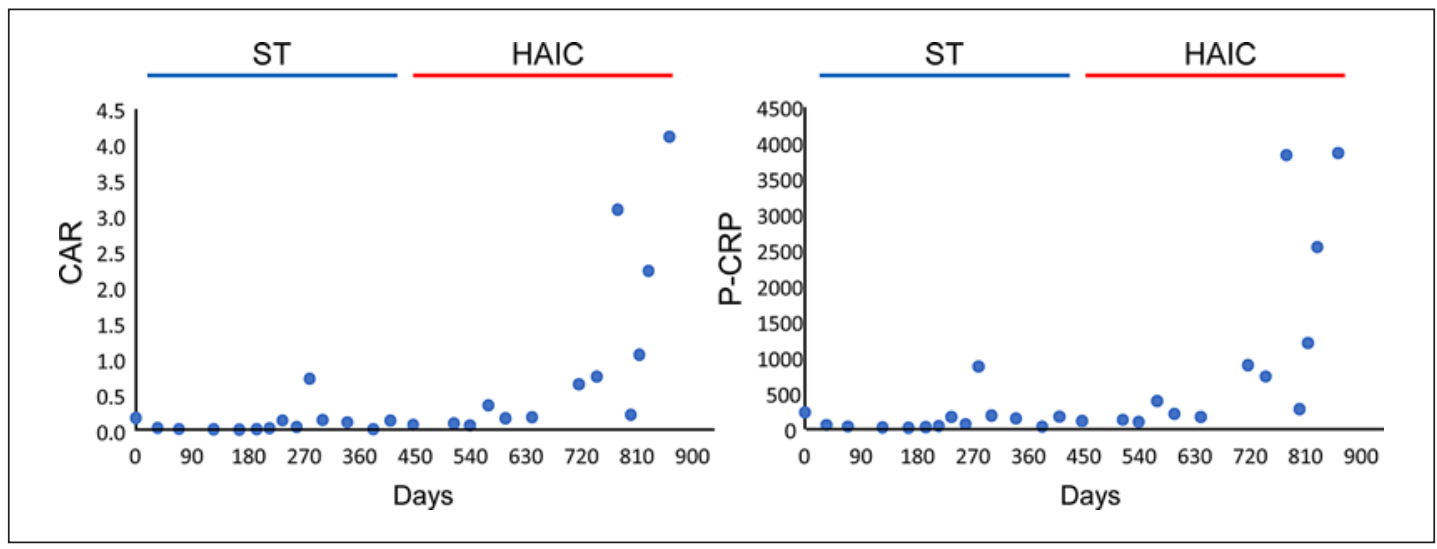

Fig. 2. Changes in SIR. Left, CAR. right, P-CRP. ST, systemic medical therapy; HAIC, hepatic arterial infusion chemotherapy; CRP/CAR, C-reactive protein/albumin ratio; P-CRP, peripheral Platelet count $\times$ C-reactive protein multiplier; SIR, systemic inflammatory response.

HAIC has been used to treat gastrointestinal malignancies with LM expecting high tumor response followed by extended survival rate with low toxicity [10-12]. However, the benefit of HAIC for BC patients with LM remains unclear although some retrospective studies reported that HAIC was effective for LM refractory to conventional systemic chemotherapy $[13,14]$.

We have presented a case of successful use of HAIC for advanced BC patients with LMs who declined ST. In addition, we observed that HAIC maintained low SIR status with a good therapeutic effect, suggesting HAIC could prolong survival in MBC patients with predominant LM via improved local control of LMs and maintaining good inflammation, nutritional, and immunologic status. HAIC may be an option for advanced BC patients with predominant LMs who cannot receive ST.

\section{Acknowledgements}

The authors thank Dr. Tyler Lahusen and Nana Muraya for helpful comments and English proofreading.

\section{Statement of Ethics}

Written ethical approval for the publication on the present case report (including images) was obtained from the husband of the patient.

\section{Conflict of Interest Statement}

All the authors declare no conflicts of interest.

\section{Funding Sources}

This work was supported in part by the Japan Society for Promotion of Science (JSPS), Grant-in-Aid for Science Research (20K17556), and JST AIP-PRISM (JPMJCR18Y5). 


\section{Case Reports in Oncology}

\begin{tabular}{|c|c|}
\hline Case Rep Oncol 2021;14:126 & 1265 \\
\hline DOI: 10.1159/000517854 & $\begin{array}{l}\text { (c) } 2021 \text { The Author(s). Published by S. Karger AG, Basel } \\
\text { www.karger.com/cro }\end{array}$ \\
\hline
\end{tabular}

Masuda et al.: Hepatic Arterial Infusion Chemotherapy for Breast Cancer with Liver Metastases

\section{Author Contributions}

T. Masuda, O. Niizeki, T. Niizeki, H. Niizeki, and K. Fujiyoshi treated the patient and provided clinical information. Y. Ando examined the imaging data. T. Masuda wrote the manuscript. K. Mimori reviewed and edited the manuscript. All authors contributed to discussions and agreed on the final version of the submitted manuscript.

\section{Data Availability Statement}

All data generated or analyzed during this study are included in this article. Further enquiries can be directed to the corresponding author.

\section{References}

1 Gordon AC, Uddin OM, Riaz A, Salem R, Lewandowski RJ. Making the case: intra-arterial therapy for less common metastases. Semin Intervent Radiol. 2017 Jun;34(2):132-9.

2 Tewes M, Peis MW, Bogner S, Theysohn JM, Reinboldt MP, Schuler M, et al. Hepatic arterial infusion chemotherapy for extensive liver metastases of breast cancer: efficacy, safety and prognostic parameters. J Cancer Res Clin Oncol. 2017 Oct;143(10):2131-41.

3 McMillan DC. Systemic inflammation, nutritional status and survival in patients with cancer. Curr Opin Clin Nutr Metab Care. 2009 May;12(3):223-6.

4 Zitvogel L, Pietrocola F, Kroemer G. Nutrition, inflammation and cancer. Nat Immunol. 2017 Jul 19;18(8): 843-50.

5 Ganeshan A, Upponi S, Hon LQ, Warakaulle D, Uberoi R. Hepatic arterial infusion of chemotherapy: the role of diagnostic and interventional radiology. Ann Oncol. 2008 May;19(5):847-51.

6 Ang C, Jhaveri K, Patel D, Gewirtz A, Seidman A, Kemeny N. Hepatic arterial infusion and systemic chemotherapy for breast cancer liver metastases. Breast J. 2013 Jan-Feb;19(1):96-9.

7 Son W, Shin SJ, Park SH, Lee SK, Park EJ, Baik SH, et al. Clinical impact of combined modified glasgow prognostic score and c-reactive protein/albumin ratio in patients with colorectal cancer. Diagnostics. 2020 Oct 22; 10(11).

8 Shishido Y, Saito H, Shimizu S, Kono Y, Murakami Y, Miyatani K, et al. Prognostic significance of platelet $\times$ c-reactive protein multiplier in patients with esophageal squamous cell carcinoma. Surg Today. 2020 Feb; 50(2):185-92.

9 Hortobagyi GN. Treatment of breast cancer. N Engl J Med. 1998 Oct 1;339(14):974-84.

10 Ko YJ, Karanicolas PJ. Hepatic arterial infusion pump chemotherapy for colorectal liver metastases: an old technology in a new era. Curr Oncol. 2014 Feb;21(1):e116-21.

11 Obi S, Sato S, Kawai T. Current status of hepatic arterial infusion chemotherapy. Liver Cancer. 2015 Sep; 4(3): 188-99.

12 Wang K, Zhang X, Wei J, Xu Y, Liu Q, Xie J, et al. Hepatic arterial infusion oxaliplatin plus oral s-1 chemotherapy in gastric cancer with unresectable liver metastases: a case series and literature review. Cancer Manag Res. 2020;12:863-70.

13 Hsiao JH, Chang HT, Tseng YD, Chiang CL, Chen IS, Chen YC, et al. Hepatic arterial infusion chemotherapy is a feasible treatment option for breast cancer with liver-predominant metastatic disease. In Vivo. 2018 Nov-Dec; 32(6):1635-41.

14 Furuta M, Watanabe J, Aramaki T, Notsu A, Yasui H. Hepatic arterial infusion chemotherapy for metastatic breast cancer patients with resistance to standard systemic chemotherapies. In Vivo. 2020 Jan-Feb;34(1): 275-82.

\section{Karger'k}

